\title{
Extrapolation procedure for low-temperature series for the square lattice spin-1 Ising model
}

\author{
I Jensen $†$ and A J Guttmann $\ddagger$ \\ Department of Mathematics, The University of Melbourne, Parkville, Victoria 3052, \\ Australia.
}

\begin{abstract}
The finite-lattice method of series expansions has been combined with a new extrapolation procedure to extend the low-temperature series for the specific heat, spontaneous magnetisation and susceptibility of the spin-1 Ising model on the square lattice. The extended series were derived by directly calculating the series to order 99 (in the variable $u=\exp \left[-J / k_{B} T\right]$ ) and using the new extrapolation procedure to calculate an additional 13-14 terms.
\end{abstract}

PACS numbers: 05.50.+q, 64.60.Fr, 02.70.-c

Short title: Extrapolation of spin-1 Ising series

August 15, 2018

$\dagger$ E-mail address: iwan@maths.mu.oz.au

$\ddagger$ E-mail address: tonyg@maths.mu.oz.au 


\section{Introduction}

In a recent paper [1] we reported on the calculation and analysis of low-temperature series for the square lattice spin-1 Ising model. The series was derived to 79th order (in the variable $u=\exp \left[-J / k_{B} T\right]$ ) using the finite-lattice method [2] and employed a new algorithm which removed much of the memory-size restrictions of previous implementations. In this paper we report on a further extension of these series to order 113 for the specific heat and spontaneous magnetisation and order 112 for the susceptibility. The extension is obtained by direct calculation of the series to order 99 and use of a new extrapolation procedure to extend the series by an additional 13 or 14 terms. The improvement in the direct series derivation is due to a more efficient implementation of the algorithm and the use of parallel computation. The extrapolation procedure is similar to and inspired by work on directed percolation [3].

\section{The series expansion technique}

The Hamiltonian defining the spin-1 Ising model in a magnetic field $h$ can be written:

$$
\mathcal{H}=J \sum_{\langle i j\rangle}\left(1-\sigma_{i} \sigma_{j}\right)+h \sum_{i}\left(1-\sigma_{i}\right)
$$

where the spin variable $\sigma_{i}=0, \pm 1$. The first sum is over nearest neighbour pairs and the second sum is over sites. The constants are chosen so the ground state $\left(\sigma_{i}=+1 \forall i\right)$ has zero energy. The low temperature expansion is based on perturbations from the fully aligned ground state. The expansion is expressed in terms of the temperature variable $u=\exp (-\beta J)$ and the field variable $\mu=\exp (-\beta h)$, where $\beta=1 / k_{B} T$. The expansion of the partition function in powers of $u$ may be expressed as

$$
Z=\sum_{n=0}^{\infty} u^{n} \Psi_{n}(\mu)
$$

where $\Psi_{n}(\mu)$ are polynomials in $\mu$. It is more convenient to express the field dependence in terms of the variable $x=1-\mu$ and truncate the expansion at $x^{2}$

$$
Z=Z_{0}(u)+x Z_{1}(u)+x^{2} Z_{2}(u)+\ldots,
$$

where $Z_{n}(u)$ is a series in $u$ formed by collecting all terms in the expansion of $Z$ containing factors of $x^{n}$. Standard definitions yield the spontaneous magnetisation

$$
M(u)=M(0)+\left.\frac{1}{\beta} \frac{\partial \ln Z}{\partial h}\right|_{h=0}=1+Z_{1}(u) / Z_{0}(u)
$$

since $x=0$ in zero field. For the zero-field susceptibility we find

$$
\chi(u)=\left.\frac{\partial M}{\partial h}\right|_{h=0}=\left.\frac{\partial}{\partial h}\left(\frac{1}{\beta Z} \frac{\partial Z}{\partial h}\right)\right|_{h=0}=\beta\left[2 \frac{Z_{2}(u)}{Z_{0}(u)}-\frac{Z_{1}(u)}{Z_{0}(u)}-\left(\frac{Z_{1}(u)}{Z_{0}(u)}\right)^{2}\right] .
$$


The specific heat series is derived from the zero field partition function (via the internal energy $\left.U=-(\partial / \partial \beta) \ln Z_{0}\right)$,

$$
C_{v}(u)=\frac{\partial U}{\partial T}=\beta^{2} \frac{\partial^{2}}{\partial \beta^{2}} \ln Z_{0}=(\beta J)^{2}\left(u \frac{\mathrm{d}}{\mathrm{d} u}\right)^{2} \ln Z_{0}(u) .
$$

So in order to obtain the series expansion of the specific heat, spontaneous magnetisation and susceptibility it suffices to calculate the three quantities $Z_{0}, Z_{1}$ and $Z_{2}$.

On the square lattice the infinite lattice partition function $Z$ can be approximated by a product of partition functions $Z_{m n}$ on finite $(m \times n)$ lattices,

$$
Z(u) \approx \prod_{m, n} Z_{m n}(u)^{a_{m n}} \quad \text { with } m \leq n \text { and } m+n \leq r,
$$

where $r$ is a cut-off which limits the size of the rectangles considered. The weights $a_{m n}$ are small integers and are known explicitly [ब] for the square lattice,

$$
a_{m n}=\left\{\begin{aligned}
1 & \text { if } m+n=r \\
-3 & \text { if } m+n=r-1 \\
3 & \text { if } m+n=r-2 \\
-1 & \text { if } m+n=r-3 \\
0 & \text { otherwise }
\end{aligned}\right.
$$

Due to the symmetry of the square lattice one obviously has that $Z_{m n}=Z_{n m}$ so one need only consider the case $m \leq n$ and change the weights $a_{m n}$ appropriately, i.e., multiply by 2 if $m<n$.

For the low-temperature expansion of the Ising model $Z_{m n}$ is calculated as the sum over all spin configurations on the finite lattice. All spins outside the $m \times n$ range are fixed at +1 . The number of terms derived correctly with the finite lattice method is given by the power of the lowest-order connected graph not contained in any of the rectangles considered, which in this case are chains of sites all in the ' 0 ' state. From the Ising Hamiltonian we see that such chains give rise to terms of order $3 r+1$. For a given value of $r$ the series expansion is thus correct to order $3 r$.

The efficient way of calculating $Z_{m n}$ is by transfer matrix techniques. We refer to [1] for a detailed description of the algorithm. For this work we used a more efficient implementation of the algorithm and a parallel computer and were able to derive the series directly up to a maximal cut-off $r_{m}=33$.

\section{Extrapolation of series}

The series can be extended significantly via an extrapolation method similar to that of [3]. Consider the series for $Z_{n}(u)$. For each $r \leq r_{m}$ we use the finite-lattice method 
to calculate the polynomials $Z_{n, r}(u)=\sum_{j=0} z_{n, j, r} u^{j}$ correct to $\mathcal{O}\left(u^{3 r+20}\right)$. As already noted these polynomials agree with the series for $Z_{n}$ to $\mathcal{O}\left(u^{3 r}\right)$. Next, we look at the sequences $d_{n, r, s}$ obtained from the difference between successive polynomials

$Z_{n, r+1}(u)-Z_{n, r}(u)=u^{3 r+1} \sum_{s \geq 0}\left(z_{n, 3 r+s+1, r+1}-z_{n, 3 r+s+1, r}\right) u^{s}=u^{3 r+1} \sum_{s \geq 0} d_{n, s, r} u^{s}$.

The first of these correction terms $d_{n, 0, r}$ is often a simple sequence which one can readily identify. In the case of $Z_{0}$ we find the sequence

$$
-d_{0,0, r}=1,2,6,18,52,138,338,778,1712, \ldots
$$

from which we conjecture

$$
d_{0,0, r}=-2^{r+2}+\left(r^{3}+3 r^{2}+2 r+18\right) / 3, \quad r \geq 2 .
$$

The formula for $d_{0,0, r}$ holds for all the $r_{m}-1$ values that we calculated and we are very confident that it is correct for all values of $r$. As was the case in [3] the higher-order correction terms $d_{n, s, r}$ can be expressed as rational functions of $d_{n, 0, r}$. Due to the form of the first correction term this leads to the general extrapolation formulae

$$
d_{n, s, r}=\frac{1}{6 s ! n !} \sum_{j=0}^{s+n+3} a_{n, s, j} r^{j}+\frac{1}{s ! n !} \sum_{j=0}^{s+n} b_{n, s, j} r^{j} 2^{r}, \quad r \geq s+2 .
$$

The factors in front of the sums have been chosen so as to make the leading coefficients particularly simple. We were able to find formulae for all correction terms up to $s=13$ for $Z_{0}$ and $Z_{1}$ and up to $s=12$ for $Z_{2}$. The coefficients in the extrapolation formulae

\begin{tabular}{|c|c|c|c|c|c|c|c|c|c|c|c|c|c|c|}
\hline \multicolumn{15}{|c|}{$a_{0,3, j}$} \\
\hline $8 / \mathrm{s}$ & 0 & 1 & 2 & 3 & 4 & 5 & 6 & 7 & 8 & 9 & 10 & 11 & 12 & 13 \\
\hline 0 & 36 & 540 & 22872 & 1107432 & 63379296 & 4055122080 & 288647444160 & 22547966414400 & 1918636884702720 & 176562491228282880 & 17472051515349043200 & 1849953059391525580800 & 208680452227780904448000 & 24984133537890061908172800 \\
\hline 1 & 4 & -388 & -15300 & -659892 & -33706248 & -1962612264 & -128643760656 & -9349955599536 & -746245210622400 & -64847088398958912 & -6093544542023399040 & -615580597302682481280 & -66523478627705920842240 & -7657040670385380368232960 \\
\hline 2 & 6 & 98 & 3708 & 148480 & 6885452 & 372299004 & 22859538096 & 1569303632880 & 119023740391728 & 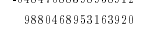 & 890825375508579936 & $\begin{array}{r}-10671272136923553248 \\
8658\end{array}$ & 9049800408163475724288 & 1009303716480382578431232 \\
\hline 3 & 2 & -8 & -350 & -15378 & -692008 & -35838744 & -2117794652 & -140423589580 & -10308461906400 & -830282122344096 & -72794312849256336 & -6901425833836745040 & -703518910546232023200 & -76730338437350841988128 \\
\hline 4 & & -2 & 12 & 850 & 39698 & 2015962 & 117161232 & 7676696540 & 556423820364 & 44185336926052 & 3818076291712376 & 356800848438240648 & 35863046515921590672 & 3858517386552205586640 \\
\hline 5 & & & 2 & -18 & -1690 & -78876 & -4420926 & -287670222 & -20887171580 & -1654723702956 & -142221175621228 & -13202390656569708 & -1317402227058041720 & -140668642000137358744 \\
\hline 6 & & & & -2 & 26 & 2956 & 132144 & 7963662 & 583444134 & 46791584450 & 4033976898388 & 373970026441792 & 37214095717203636 & 3959799742297856756 \\
\hline 7 & & & & & 2 & -36 & -4728 & -195000 & -12264582 & -1033287048 & -91299422798 & -8506311905850 & -846366898168088 & -89980514374296832 \\
\hline 8 & & & & & & -2 & 48 & 7080 & 258576 & 16303470 & 1658091108 & 161206833194 & 16060785342426 & 1705608425121458 \\
\hline 9 & & & & & & & 2 & -62 & -10080 & -309132 & -18208734 & -2480840274 & -265232878130 & -27784108335412 \\
\hline 10 & & & & & & & & -2 & 78 & 13790 & 327676 & 15045162 & 3551877714 & 416896760852 \\
\hline 11 & & & & & & & & & 2 & -96 & -18266 & -289710 & -2605714 & -4993549652 \\
\hline 12 & & & & & & & & & & -2 & 116 & 23558 & 165102 & -24785696 \\
\hline 13 & & & & & & & & & & & 2 & -138 & -29710 & 81916 \\
\hline 14 & & & & & & & & & & & & -2 & 162 & 36760 \\
\hline 15 & & & & & & & & & & & & & 2 & -188 \\
\hline 16 & & & & & & & & & & & & & & -2 \\
\hline \multicolumn{15}{|c|}{$b_{0, s, j}$} \\
\hline$\overline{s / j}$ & 0 & 1 & 2 & 3 & 4 & 5 & 6 & 7 & 8 & 9 & 10 & 11 & 12 & 13 \\
\hline 0 & -4 & -8 & -26 & -174 & -1794 & -29040 & -621855 & -16476705 & -470890980 & -14775299910 & -502882151625 & -18429217173675 & -734477218900425 & -31757793382715400 \\
\hline 1 & & 4 & 10 & -70 & -863 & -13364 & $-218665 \frac{1}{2}$ & $-3099628 \frac{1}{2}$ & $-50245171 \frac{1}{2}$ & -1002068919 & $-25366148178 \frac{3}{4}$ & $-911394577226 \frac{1}{4}$ & $-40664486659736 \frac{1}{4}$ & $-1919833204276777 \frac{1}{2}$ \\
\hline 2 & & & -4 & -6 & 409 & 4105 & 43289 & 618625 & $-275468 \frac{2}{4}$ & -186136038 & $-6579087314 \frac{4}{8}$ & $-231588134427 \frac{4}{8}$ & $-6538249560737_{4}^{4}$ & $\begin{array}{r}-170426278459944 \\
-2\end{array}$ \\
\hline 3 & & & & 4 & -4 & -1085 & $-8407 \frac{1}{2}$ & 11116 & $819836 \frac{1}{2}$ & $77789387^{\frac{3}{4}}$ & $2571738900 \frac{5}{8}$ & $58200571408 \frac{8}{8}$ & $1572709594180 \frac{\frac{1}{16}}{16}$ & $32784296682271 \frac{7}{8}$ \\
\hline 4 & & & & & -4 & 20 & 2165 & $8942 \frac{1}{2}$ & $-429182 \frac{2}{4}$ & -8136639 & $-322113948 \frac{8}{8}$ & $-8743272683 \frac{8}{\frac{3}{4}}$ & $-73717696634 \frac{16}{\frac{16}{6}}$ & $687212762691 \frac{18}{16}$ \\
\hline 5 & & & & & & 4 & -42 & $\begin{array}{r}-3689 \\
-2\end{array}$ & 4144 & $1736096 \frac{1}{4}$ & $23447878 \frac{8}{8}$ & $610009090 \frac{4}{8}$ & $12227448244 \frac{11}{16}$ & $-584353065430 \frac{16}{11}$ \\
\hline 6 & & & & & & & -4 & 70 & 5670 & -46872 & $-4727903 \frac{8}{4}$ & $-35390845 \frac{8}{\frac{8}{2}}$ & $-8870615 \frac{3}{4}$ & $13285766431 \frac{7}{16}$ \\
\hline 7 & & & & & & & & 4 & -104 & -8094 & 142365 & $10435103 \frac{\bar{\pi}}{4}$ & $-3532691 \frac{1}{4}$ & $-4219620181 \frac{9}{16}$ \\
\hline 8 & & & & & & & & & -4 & 144 & 10920 & -321585 & $-20006175 \frac{3}{4}$ & $213929108 \frac{1}{4}$ \\
\hline 9 & & & & & & & & & & 4 & -190 & -14080 & 623865 & $34494495 \frac{4}{4}$ \\
\hline 10 & & & & & & & & & & & -4 & 242 & 17479 & -1097239 \\
\hline 11 & & & & & & & & & & & & 4 & -300 & -20995 \\
\hline 12 & & & & & & & & & & & & & -4 & 364 \\
\hline 13 & & & & & & & & & & & & & & 4 \\
\hline
\end{tabular}
are listed in tables 13 .

Table 1. Coefficients $a_{0, s, j}$ and $b_{0, s, j}$ in the extrapolation formula (11) for $Z_{0}$. 
Table 2. Coefficients $a_{1, s, j}$ and $b_{1, s, j}$ in the extrapolation formula (11) for $Z_{1}$.

\begin{tabular}{|c|c|c|c|c|c|c|c|c|c|c|c|c|c|c|}
\hline \multicolumn{15}{|c|}{$a_{1, s, j}$} \\
\hline$s / 1$ & 0 & 1 & 2 & 3 & 4 & 5 & 6 & 7 & 8 & 9 & 10 & 11 & 12 & 13 \\
\hline 0 & & $\begin{array}{r}-1008 \\
242\end{array}$ & $\begin{aligned}-76272 \\
26776\end{aligned}$ & $\begin{array}{r}-5684112 \\
2132988\end{array}$ & $\begin{array}{r}-457173792 \\
169186080\end{array}$ & $\begin{array}{r}-39103403040 \\
14031817536\end{array}$ & $\begin{array}{r}-3574330130880 \\
1234927394784\end{array}$ & $\begin{array}{r}-348267251263680 \\
115716608013024\end{array}$ & $\begin{array}{l}-36106919860838400 \\
1154417471675068\end{array}$ & -3997322177228016112800 & -462907224587766067200 & -56960009225508461688422400 & 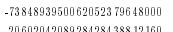 & $\begin{array}{l}-100655747859870107990083584400 \\
\text { - }\end{array}$ \\
\hline & $\begin{array}{l}-10 \\
-13\end{array}$ & $\begin{array}{l}242 \\
161\end{array}$ & $\begin{array}{r}26776 \\
3890\end{array}$ & $\begin{array}{r}2132988 \\
-11104\end{array}$ & $\begin{array}{l}169186080 \\
-10362700\end{array}$ & $\begin{array}{l}14031817536 \\
-1228736780\end{array}$ & $\begin{array}{l}1234927394784 \\
-124161954720\end{array}$ & $\begin{array}{l}115716608013024 \\
-12398829627432\end{array}$ & $\begin{array}{l}\begin{array}{l}115441747167559688 \\
-1273546890227328\end{array} \\
\text {. }\end{array}$ & $\begin{array}{r}1224242942187936384 \\
-136597270576055328\end{array}$ & $\begin{array}{l}137714989912773327360 \\
-15386179110821861184\end{array}$ & $\begin{array}{l}1639963998645157144435200 \\
-1823326122928804380864\end{array}$ & $\begin{array}{l}\begin{array}{l}2060202042089284284438812160 \\
-227313813910382067927168\end{array} \\
-\end{array}$ & $\begin{array}{l}272692487745604926720576000 \\
-2978610602558157422698880\end{array}$ \\
\hline 3 & -5 & -76 & -2693 & -88837 & -3049540 & -100973728 & -1890947308 & 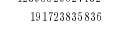 & 407500324058580 & 5702911364579304 & 735898260522888000 & 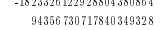 & 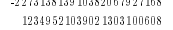 & 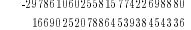 \\
\hline 4 & -2 & 7 & 312 & 12959 & 526035 & 23570715 & 1144281164 & 57449415410 & 2729697798164 & 90106090294292 & -4306747906782568 & -1641298736512799072 & -3004891549031717050008 & -4809401010622328646550800 \\
\hline 5 & & & -11 & -793 & -35251 & -1674878 & -88419027 & -5114559137 & -315077153986 & -20142410271322 & - 1282219400735740 & -73711506188062096 & -2454291894283312912 & 302424204333008932640 \\
\hline 6 & & & & & 1611 & 71790 & 3837282 & 232243641 & 15355331735 & 1081711840093 & 80200154284766 & 6155735350915480 & 47559006601882899368 & 34934157032467155320 \\
\hline 7 & & & & & & -2852 & -121842 & -7085208 & -490136073 & -36372405428 & -2855093133545 & -236164037257269 & -20420107101284544372 & -18196941976689399020 \\
\hline 8 & & & & & & 35 & 4596 & 180960 & 11069628 & 891555753 & 73516462976 & 6324527102839 & 572875721359139 & 54403254827920427 \\
\hline 9 & & & & & & 2 & -47 & -6917 & -240352 & -14817384 & -1460017005 & -132977895083 & -12335475680011 & - 1208966177416998 \\
\hline 10 & & & & & & & -2 & 61 & 9883 & 286375 & 16521220 & 2223262547 & 222732548753 & 21808039004180 \\
\hline 11 & & & & & & & & 2 & -77 & -13556 & -300155 & -13327961 & -3238529375 & -355244044168 \\
\hline 12 & & & & & & & & & -2 & 95 & 17992 & 257333 & 1130371 & 4637002786 \\
\hline 13 & & & & & & & & & & 2 & -115 & -23241 & - 127937 & 25630356 \\
\hline 14 & & & & & & & & & & & -2 & 137 & 29347 & -123620 \\
\hline 15 & & & & & & & & & & & & 2 & -161 & -36348 \\
\hline 16 & & & & & & & & & & & & & -2 & 187 \\
\hline 17 & & & & & & & & & & & & & & \\
\hline \multicolumn{15}{|c|}{$\frac{b_{1, n, j}}{b_{1}}$} \\
\hline $8 / 1 / 5$ & 0 & 1 & 2 & 3 & 4 & 5 & 6 & 7 & 8 & 9 & 10 & 11 & 12 & 13 \\
\hline 0 & 0 & 4 & 38 & 669 & 14274 & 382185 & 11367745 & 374646825 & 13414424940 & 521931313260 & 21950041038975 & 994043048211675 & 48323690206458975 & $2511969014601108562 \frac{1}{2}$ \\
\hline & 4 & 8 & 28 & 294 & 3154 & 45380 & $6.59431 \frac{1}{2}$ & $6912974 \frac{1}{4}$ & -63875241 & -95160599361 & $-5776389937698 \frac{8}{4}$ & $-30629467750426 \frac{2}{\frac{2}{8}}$ & $-15983499663491021 \frac{1}{4}$ & $-8584441586665222005 \frac{\frac{5}{8}}{8}$ \\
\hline 2 & & -4 & -10 & 52 & 245 & 6049 & $130525 \frac{1}{2}$ & $2055016 \frac{3}{4}$ & $70181556 \frac{1}{2}$ & $24432256322 \frac{2}{4}$ & $87983829793 \frac{1}{3}$ & $3603111185211 \frac{8}{16}$ & $151803540464029 \frac{4}{8}$ & $66973007607695555 \frac{8}{16}$ \\
\hline 3 & & & 4 & 6 & -365 & -2595 & $-32166 \frac{1}{2}$ & $-703727 \frac{1}{2}$ & $-4071664 \frac{1}{4}$ & -104301900 & $-4278680737 \frac{8}{4}$ & -98042635537 & $-4951488221400 \frac{8}{4}$ & $-2031206705981106 \frac{6}{3}$ \\
\hline 4 & & & & -4 & 4 & 1005 & $5647 \frac{2}{2}$ & $-6373 \frac{2}{2}$ & $428855 \frac{1}{2}$ & $-49051827 \frac{1}{2}$ & -1421306965 & $-22978129383 \frac{11}{16}$ & $-986226770316_{16}^{4}$ & $-229749043272411^{\frac{3}{4}}$ \\
\hline 5 & & & & & 4 & -20 & $\begin{array}{c}-2039 \\
-203\end{array}$ & $-4700 \frac{1}{2}$ & $356788 \frac{2}{4}$ & 3508470 & $251750258 \frac{8}{4}$ & 6762309950 & $33331027784 \frac{16}{116}$ & $1317242138953 \frac{3}{16}$ \\
\hline 6 & & & & & & -4 & 42 & 3507 & -9884 & $-1495436 \frac{1}{4}$ & $-11750983 \frac{1}{8}$ & $-5485053311 \frac{1}{4}$ & $-13280506010 \frac{16}{16}$ & $4846390075867_{\frac{3}{3}}^{\frac{4}{3}}$ \\
\hline 7 & & & & & & & 4 & -70 & -5422 & 53820 & $4151708 \frac{1}{4}$ & $117846225_{\frac{3}{8}}^{\frac{3}{3}}$ & $2343178647 \frac{16}{76}$ & $4806055084 \frac{3}{16}$ \\
\hline 8 & & & & & & & & -4 & 104 & 7770 & -149835 & $-9271523 \frac{1}{4}$ & $\begin{array}{l}43741046 \frac{16}{4} \\
4\end{array}$ & $2899134157 \frac{6}{16}$ \\
\hline 9 & & & & & & & & & 4 & -144 & -10510 & 328405 & $17901765 \frac{3}{4}$ & $-272545523 \frac{1}{4}$ \\
\hline 10 & & & & & & & & & & -4 & 190 & 13574 & -628287 & $-30979126 \frac{3}{4}$ \\
\hline 11 & & & & & & & & & & & 4 & -242 & -16867 & 1096849 \\
\hline 12 & & & & & & & & & & & & -4 & 300 & 20267 \\
\hline 13 & & & & & & & & & & & & & 4 & -364 \\
\hline 14 & & & & & & & & & & & & & & \\
\hline
\end{tabular}

Table 3. Coefficients $a_{2, s, j}$ and $b_{2, s, j}$ in the extrapolation formula (11) for $Z_{2}$.

\begin{tabular}{|c|c|c|c|c|c|c|c|c|c|c|c|c|c|}
\hline \multicolumn{14}{|c|}{$a_{2, s, j}$} \\
\hline$s / j$ & 0 & 1 & 2 & 3 & 4 & 5 & 6 & 7 & 8 & 9 & 10 & 11 & 12 \\
\hline 0 & 0 & 960 & 199008 & 25859664 & 3105873216 & 366981638400 & 43962925841280 & 5412646450270080 & 689859653042488320 & 91351020237486105600 & 12589478624473041715200 & 1806730592586035116492800 & 269979775259474769527808000 \\
\hline 1 & 12 & 724 & 3872 & -3918624 & -664081632 & -88392730560 & -11084244867072 & -1384694987443488 & -176331199274687232 & -23140865178023786496 & -3146715137132668707840 & -444530245545400234049280 & -65310818282764528381701120 \\
\hline 2 & -20 & -444 & -34224 & -1984608 & -100703480 & -3423052408 & 152494743744 & 58829587491792 & 10515670803566400 & 1629900454944139200 & 243735746832642107520 & 36500387580624231020736 & 5565229584050566554128640 \\
\hline 3 & 16 & -48 & 1788 & 325744 & 27280764 & 2042841660 & 148133624112 & 10422978961984 & 671732365773200 & 31014499461745399 & -1105330916217243648 & -683352705387601823616 & -155665991250042317339904 \\
\hline 4 & 2 & 62 & 2056 & 49792 & 547580 & -62926964 & -9069431144 & -924884356296 & -87052546757280 & -7980855749069280 & -715388251788131360 & -60821071543388792752 & - 4414115889872814761376 \\
\hline 5 & 2 & -4 & -286 & -11426 & -409240 & -14714408 & -466639676 & -4246070624 & 1552859277500 & 259271668545748 & 32421044232235536 & 3730895758411050000 & 413972960682773319120 \\
\hline 6 & & -2 & 8 & 754 & 32534 & 1427150 & 66602412 & 3204308732 & 144793941316 & 4584284710972 & -173506172385408 & -64646756919141120 & -10662860072358795800 \\
\hline 7 & & & 2 & -14 & -1558 & -67700 & -3405654 & - 188754426 & -11056264376 & -654356019664 & -36883161160012 & -1658325705468872 & 1972409671354252 \\
\hline 8 & & & & -2 & 22 & 2784 & 116304 & 6427038 & 414871986 & 28039294862 & 1943327703680 & 135094260867632 & 8967575039268340 \\
\hline 9 & & & & & 2 & -32 & -4512 & -174028 & -10163570 & -774000120 & -58982092862 & -4593416253250 & - 365367972622928 \\
\hline 10 & & & & & & -2 & 44 & 6816 & 232224 & 13674990 & 1290331376 & 109505894322 & 9324373410550 \\
\hline 11 & & & & & & & 2 & -58 & -9764 & -277408 & -15198942 & -1993340778 & -186906331802 \\
\hline 12 & & & & & & & & -2 & 74 & 13418 & 290880 & 11939322 & 2942846678 \\
\hline 13 & & & & & & & & & 2 & -92 & -17834 & -248470 & 141926 \\
\hline 14 & & & & & & & & & & -2 & 112 & 23062 & 120410 \\
\hline 15 & & & & & & & & & & & 2 & -134 & -29146 \\
\hline 16 & & & & & & & & & & & & -2 & 158 \\
\hline 17 & & & & & & & & & & & & & 2 \\
\hline \multicolumn{14}{|c|}{$b_{2,3, j}$} \\
\hline$s / \mathrm{j}$ & 0 & 1 & 2 & 3 & 4 & 5 & 6 & 7 & 8 & 9 & 10 & 11 & 12 \\
\hline 0 & 0 & 0 & -40 & -1848 & -72579 & -2888655 & -118290915 & -5121342765 & -235010259540 & -11456914728105 & -592786074379275 & -32513880609579600 & $-1887019647180154987 \frac{1}{2}$ \\
\hline 1 & 4 & 0 & .50 & -1176 & -26112 & -647905 & $-16453117 \frac{1}{2}$ & $-425308222 \frac{1}{2}$ & $-10281313150 \frac{1}{2}$ & $-181320757591 \frac{1}{2}$ & 2606202716040 & $622231248666307 \frac{1}{2}$ & $56560097077675104 \frac{2}{\frac{3}{6}}$ \\
\hline 2 & -4 & -12 & -40 & -344 & -3513 & -44366 & $-486457 \frac{2}{2}$ & $-878527 \frac{2}{2}$ & $\begin{array}{l}105956879 \\
2\end{array}$ & 996828993 & -302888778255 & $-35962386099941 \frac{2}{4}$ & $-2973976984644465 \frac{8}{16}$ \\
\hline 3 & & 4 & 14 & -28 & -36 & -3009 & $-85659 \frac{1}{2}$ & -1339020 & $-70112132 \frac{3}{4}$ & -2289397563 & $-61537586981 \frac{5}{8}$ & $-1417645036817 \frac{1}{2}$ & 14046475617195 \\
\hline 4 & & & -4 & -10 & 325 & 2170 & $29241 \frac{1}{2}$ & 736029 & $5619955 \frac{4}{4}$ & $250213920 \frac{1}{4}$ & 8870308461 & $182363269816 \frac{2}{4}$ & 5892713122080 \\
\hline 5 & & & & 4 & 0 & -945 & $-5052 \frac{1}{2}$ & $-5841 \frac{1}{2}$ & $-1080203 \frac{4}{4}$ & $34632655 \frac{4}{4}$ & 705890175 & 1850093234 & $713236878450 \frac{1}{4}$ \\
\hline 6 & & & & & -4 & 16 & 1955 & $4147 \frac{1}{2}$ & $-292766 \frac{1}{4}$ & $-1058909 \frac{1}{4}$ & $-217483272 \frac{1}{2}$ & $-5310678979 \frac{3}{8}$ & $-1036978067 \frac{1}{6}$ \\
\hline 7 & & & & & & 4 & -38 & -3395 & 9954 & $1310900 \frac{1}{4}$ & $5720061 \frac{2}{8}$ & $539675633^{\frac{3}{4}}$ & $13147234615 \frac{6}{8}$ \\
\hline 8 & & & & & & & -4 & 66 & 5278 & $-5264^{4}$ & $-3735338 \frac{1}{4}$ & $-343039 \frac{1}{8}$ & $-464689558 \frac{8}{16}$ \\
\hline 9 & & & & & & & & 4 & -100 & -7590 & 146385 & $8459448 \frac{8}{4}$ & $-61062655 \frac{1}{2}$ \\
\hline 10 & & & & & & & & & -4 & 140 & 10290 & -321145 & $-16469070 \frac{2}{4}$ \\
\hline 11 & & & & & & & & & & 4 & -186 & -13310 & 615230 \\
\hline 12 & & & & & & & & & & & -4 & 238 & 16555 \\
\hline 13 & & & & & & & & & & & & 4 & -296 \\
\hline 14 & & & & & & & & & & & & & -4 \\
\hline
\end{tabular}


It is clear from equation (11) that the $r_{m}-s-2$ terms available from the various sequences for the correction terms are not sufficient to determine all the $2(s+n+2)+1$ unknown coefficients in the extrapolation formulae for large $s$. However, from tables 10 we immediately see that the leading coeffients, $a_{n, s, s+n+3}$ and $b_{n, s, s+n}$, in the extrapolation formulae are alternating in sign but otherwise constant, e.g., $a_{n, s, s+n+3}=(-1)^{s+n} 2$ and $b_{n, s, s+n}=-(-1)^{s+n} 4$. In general we have found that the leading coeffients are expressible as polynomials in $s$,

$$
a_{n, s, s+n+3-k}=\frac{(-1)^{s+n}}{(2 s) !} \sum_{j=0}^{2 k+l} \alpha_{n, k, j} s^{j},
$$

and

$$
b_{n, s, s+n-k}=\frac{(-1)^{s+n}}{(s+1) ! 4^{s-1}} \sum_{j=0}^{2 k} \beta_{n, k, j} s^{j},
$$

where $l=\max (k-3,0)$. The coefficients of these polynomials are listed in table $₫$ and table 5 .

This time we note that the 2 or 3 leading coefficients are independent of $n$. And indeed we find that $\beta_{n, k, 2 k-j} / 3^{k}$ is a polynomial in $k$ of order $2 j+1$. In particular we have,

$$
\begin{gathered}
\beta_{n, k, 2 k}=-3^{k}(k+1), \\
\beta_{n, k, 2 k-1}=3^{k}\left(278 k^{3}+99 k^{2}-179 k\right) / 27, \\
\beta_{n, k, 2 k-2}=\left\{\begin{array}{l}
3^{k}\left(77284 k^{5}-233636 k^{4}+145247 k^{3}+233636 k^{2}-222531 k\right) / 1458 \quad n=0 \\
3^{k}\left(77284 k^{5}-233636 k^{4}+148487 k^{3}+233636 k^{2}-225771 k\right) / 1458 \quad n=1 \\
3^{k}\left(77284 k^{5}-233636 k^{4}+151727 k^{3}+231692 k^{2}-230955 k\right) / 1458 \quad n=2,
\end{array}\right.
\end{gathered}
$$

and

$$
\beta_{n, k, 2 k-3}=\left\{\begin{array}{r}
3^{k}\left(107424760 k^{7}-981604100 k^{6}+3689847622 k^{5}-5987330165 k^{4}\right. \\
\left.+1103673490 k^{3}+6968934265 k^{2}-4900945872 k\right) / 590490 \\
3^{k}\left(107424760 k^{7}-981604100 k^{6}+3703358422 k^{5}-6035726045 k^{4}\right. \\
\left.+1135273210 k^{3}+7017330145 k^{2}-4946056392 k\right) / 590490 \\
3^{k}\left(107424760 k^{7}-981604100 k^{6}+3716869222 k^{5}-6092228405 k^{4}\right. \\
\left.+1180199050 k^{3}+7073832505 k^{2}-5004493032 k\right) / 590490 \quad n=2 .
\end{array}\right.
$$

So when calculating the extrapolation formulae (11) we first used the sequences for the correction terms to predict as many polynomials as possible. When we ran out of terms we then predicted as many of the leading coefficients from (12) and (13) as possible. This in turn allowed us to find more extrapolation formulae, which we could use (together with the formulae for $\beta_{n, k, 2 k-j}$ ) to find more of the formulas for the leading 
Table 4. The coefficients $\alpha_{n, k, j}$ in the extrapolation formulae (12).

\begin{tabular}{|c|c|c|c|c|c|c|}
\hline $\mathrm{k} / \mathrm{j}$ & 0 & 1 & 2 & 3 & 4 & 5 \\
\hline \multicolumn{7}{|c|}{$\alpha_{0, k, j}$} \\
\hline 0 & 2 & 12 & 96 & 25920 & 0 & 0 \\
\hline 1 & & 2 & -1468 & 510840 & 1300549152 & 6977845103040 \\
\hline 2 & & 2 & -558 & -888576 & -2703028424 & -15961597601040 \\
\hline 3 & & & -428 & 600150 & 1803808536 & 12990760770024 \\
\hline 4 & & & 6 & 38850 & -409368974 & -4669644914724 \\
\hline 5 & & & & -7950 & -18927216 & 667412568090 \\
\hline 6 & & & & 126 & 5412484 & 519181770 \\
\hline 7 & & & & & -224616 & -5708659068 \\
\hline 8 & & & & & 6354 & 428756328 \\
\hline 9 & & & & & -96 & -15678630 \\
\hline 10 & & & & & & 484770 \\
\hline 11 & & & & & & -10656 \\
\hline 12 & & & & & & 96 \\
\hline \multicolumn{7}{|c|}{$\alpha_{1, k, j}$} \\
\hline 0 & 2 & 10 & 312 & 7200 & 967680 & 0 \\
\hline 1 & & 2 & -1192 & 566700 & 1554817152 & 8201223021600 \\
\hline 2 & & 2 & -522 & -1082526 & -3148467656 & -18452430302760 \\
\hline 3 & & & -428 & 592890 & 2028534576 & 14630011358544 \\
\hline 4 & & & 6 & 39480 & -411219494 & -5057308922574 \\
\hline 5 & & & & -7950 & -20088936 & 678397444650 \\
\hline 6 & & & & 126 & 5432476 & 1825419990 \\
\hline 7 & & & & & -224616 & -5791418388 \\
\hline 8 & & & & & 6354 & 430798878 \\
\hline 9 & & & & & -96 & -15704550 \\
\hline 10 & & & & & & 484770 \\
\hline 11 & & & & & & -10656 \\
\hline 12 & & & & & & 96 \\
\hline \multicolumn{7}{|c|}{$\alpha_{2, k, j}$} \\
\hline 0 & 2 & 4 & 384 & -14400 & 483840 & 0 \\
\hline 1 & & 2 & -940 & 679560 & 1809266592 & 9448276380480 \\
\hline 2 & & 2 & -510 & -1261176 & -3582329672 & -20938915839600 \\
\hline 3 & & & -428 & 598470 & 2221265016 & 16212179478024 \\
\hline 4 & & & 6 & 39930 & -415205294 & -5412395679564 \\
\hline 5 & & & & -7950 & -20805456 & 691017125850 \\
\hline 6 & & & & 126 & 5445412 & 2648223930 \\
\hline 7 & & & & & -224616 & -5853962268 \\
\hline 8 & & & & & 6354 & 432269568 \\
\hline 9 & & & & & -96 & -15721830 \\
\hline 10 & & & & & & 484770 \\
\hline 11 & & & & & & -10656 \\
\hline 12 & & & & & & 96 \\
\hline
\end{tabular}


Table 5. The coefficients $\beta_{n, k, j}$ in the extrapolation formulae (13).

\begin{tabular}{|c|c|c|c|c|c|c|c|c|c|}
\hline $\mathrm{k} / \mathrm{j}$ & 0 & 1 & 2 & 3 & 4 & 5 & 6 & 7 & 8 \\
\hline \multicolumn{10}{|c|}{$\beta_{0, k, j}$} \\
\hline 0 & -1 & 0 & 0 & 0 & 0 & 0 & 0 & 0 & 0 \\
\hline 1 & & 22 & 1658 & 334656 & 153601904 & 109900869120 & 119835268440320 & 179701691558215680 & 358810347942675191808 \\
\hline 2 & & -6 & -2385 & -649384 & $-348301926 \frac{2}{3}$ & -276393269472 & -325054574102944 & -517144503241342976 & $-1083429067670151410841 \frac{3}{5}$ \\
\hline 3 & & & 754 & 403500 & 284418460 & 266027954552 & $350188538012687 \frac{1}{9}$ & 606338380910234368 & 1357922016436272064896 \\
\hline 4 & & & -27 & -96524 & $-108269551 \frac{2}{3}$ & -129814389840 & -200292896954500 & $-388885779063580337 \frac{7}{9}$ & $-950703086097155610426 \frac{2}{3}$ \\
\hline 5 & & & & 7860 & 20243976 & 35294435450 & $67835150491952 \frac{2}{3}$ & $153240568087470602 \frac{2}{3}$ & 419124576286737422400 \\
\hline 6 & & & & -108 & $-1748436 \frac{2}{3}$ & -5461778850 & -14237073600841 & $-39235294734473786 \frac{3}{3}$ & $-123701080180932859946 \frac{2}{15}$ \\
\hline 7 & & & & & 55980 & 465582548 & $1869074318751 \frac{1}{3}$ & 6694588679545816 & 25258034274069075192 \\
\hline 8 & & & & & -405 & -19729020 & -150316130097 & $-764582679410893 \frac{1}{3}$ & -3623005587850488121 \\
\hline 9 & & & & & & 326970 & $6996149842 \frac{8}{9}$ & 57616149115192 & 365799796385163936 \\
\hline 10 & & & & & & -1458 & -168308595 & $-2763401680710 \frac{2}{9}$ & $-25727820621001866 \frac{14}{15}$ \\
\hline 11 & & & & & & & 1688526 & $78924402909 \frac{1}{3}$ & 1230228773135952 \\
\hline 12 & & & & & & & -5103 & -1196493480 & $-38326543230336 \frac{2}{3}$ \\
\hline 13 & & & & & & & & 8015112 & 725790362976 \\
\hline 14 & & & & & & & & -17496 & -7483535892 \\
\hline 15 & & & & & & & & & 35779320 \\
\hline 16 & & & & & & & & & -59049 \\
\hline \multicolumn{10}{|c|}{$\beta_{1, k, j}$} \\
\hline 0 & -1 & 0 & 0 & 0 & 0 & 0 & 0 & 0 & 0 \\
\hline 1 & & 22 & 1874 & 380544 & 173844464 & 124703585280 & 135892573287680 & 203891746284011520 & 407060480146447669248 \\
\hline 2 & & -6 & -2505 & -717448 & $-387796806 \frac{2}{3}$ & -309968480352 & -365371396244512 & -5826263556825555904 & $-1221984137726510908569 \frac{3}{5}$ \\
\hline 3 & & & 754 & 427116 & 309285900 & 293508255992 & $388934364085487 \frac{1}{9}$ & 676720632830112000 & 1519953696088914094464 \\
\hline 4 & & & -27 & -97964 & $-114431071 \frac{2}{3}$ & -140364933360 & -219200100776580 & $-429068174740539313 \frac{7}{9}$ & $-1054354166560941235770 \frac{2}{3}$ \\
\hline 5 & & & & 7860 & 20801176 & 37316508410 & $72997883541272 \frac{2}{3}$ & $166848798285151882 \frac{2}{3}$ & 459887247973724918464 \\
\hline 6 & & & & -108 & $-1759236 \frac{2}{3}$ & -5648008290 & -15047082876273 & $-42102339808215418 \frac{3}{3}$ & $-134133831039985827818 \frac{2}{15}$ \\
\hline 7 & & & & & 55980 & 472540628 & $1940426816751 \frac{1}{3}$ & 7075210989955416 & 27042955126506979800 \\
\hline 8 & & & & & -405 & -19793820 & -153559531937 & $-795936397412877 \frac{1}{3}$ & -3828497810715795865 \\
\hline 9 & & & & & & 326970 & $7058663482 \frac{8}{9}$ & 59143290605752 & 381544301457679104 \\
\hline 10 & & & & & & -1458 & -168648795 & $-2803243801350 \frac{2}{9}$ & $-26506146752612586 \frac{14}{15}$ \\
\hline 11 & & & & & & & 1688526 & $79382817117 \frac{1}{3}$ & 1253639579258096 \\
\hline 12 & & & & & & & -5103 & -1198126440 & $-38712986171808 \frac{2}{3}$ \\
\hline 13 & & & & & & & & 8015112 & 728717117184 \\
\hline 14 & & & & & & & & -17496 & -7490884212 \\
\hline 15 & & & & & & & & & 35779320 \\
\hline 16 & & & & & & & & & -59049 \\
\hline \multicolumn{10}{|c|}{$\beta_{2, k, j}$} \\
\hline 0 & -1 & 8 & 0 & 0 & 0 & 0 & 0 & 0 & 0 \\
\hline 1 & & 22 & 1826 & 399904 & 188173424 & 136763942784 & 150135943911680 & 226399980054487040 & 453481872936027199488 \\
\hline 2 & & -6 & -2553 & -747352 & $-415492486 \frac{2}{3}$ & -337028036672 & -400806757117984 & -643091153929042944 & $-1354435396148323267737 \frac{3}{5}$ \\
\hline 3 & & & 754 & 438668 & 326540300 & 315331137752 & $422565255743151 \frac{1}{9}$ & $741047725232481564 \frac{4}{9}$ & 1673542368950203659648 \\
\hline 4 & & & -27 & -98972 & $-118710111 \frac{2}{3}$ & -148615548440 & -235371247103300 & $-465345164498565169 \frac{7}{9}$ & $-1151609375547099787322 \frac{2}{3}$ \\
\hline 5 & & & & 7860 & 21201176 & 38883012026 & $77347956643752 \frac{2}{3}$ & $178970258611925237 \frac{1}{3}$ & 497699046774849639872 \\
\hline 6 & & & & -108 & $-1767876 \frac{2}{3}$ & -5793139250 & -15721316702945 & $-44622273568721818 \frac{3}{3}$ & $-143694733990288250474 \frac{2}{15}$ \\
\hline 7 & & & & & 55980 & 478155188 & $1999479461647 \frac{1}{3}$ & $7405891926422253 \frac{1}{3}$ & 28659202377280586328 \\
\hline 8 & & & & & -405 & -19848900 & -156259593217 & $-822961941093677 \frac{1}{3}$ & -4012551877454570521 \\
\hline 9 & & & & & & 326970 & $7112021962 \frac{8}{9}$ & $60457289130459 \frac{5}{9}$ & 395524266443898240 \\
\hline 10 & & & & & & -1458 & -168948171 & $-2837772622950 \frac{2}{9}$ & $-27193733020204138 \frac{14}{15}$ \\
\hline 11 & & & & & & & 1688526 & $79787198493 \frac{1}{3}$ & 1274324332887664 \\
\hline 12 & & & & & & & -5103 & -1199596104 & $-39056845236000 \frac{2}{3}$ \\
\hline 13 & & & & & & & & 8015112 & 731355327360 \\
\hline 14 & & & & & & & & -17496 & -7497602676 \\
\hline 15 & & & & & & & & & 35779320 \\
\hline 16 & & & & & & & & & -59049 \\
\hline
\end{tabular}


coefficients $a_{n, s, s+n+3-k}$ and $b_{n, s, s+n-k}$. We repeat this until the process stopped with the extrapolation formulae listed above.

From $Z_{n, 33}(u)$ we extended the series for $Z_{0}$ and $Z_{1}$ to $\mathcal{O}\left(u^{113}\right)$, while the series for $Z_{2}$ was extended to $\mathcal{O}\left(u^{112}\right)$. The resulting new low-temperature series terms are listed in table 6. The series terms for $n<80$ can be found in [1]. The full series is also available by electronic mail or via the world wide web (see end of article for details).

Table 6. New low-temperature series terms for the square lattice spin-1 Ising magnetisation, $M(u)$, susceptibility, $\chi(u)$, and specific heat, $C_{v}(u)$.

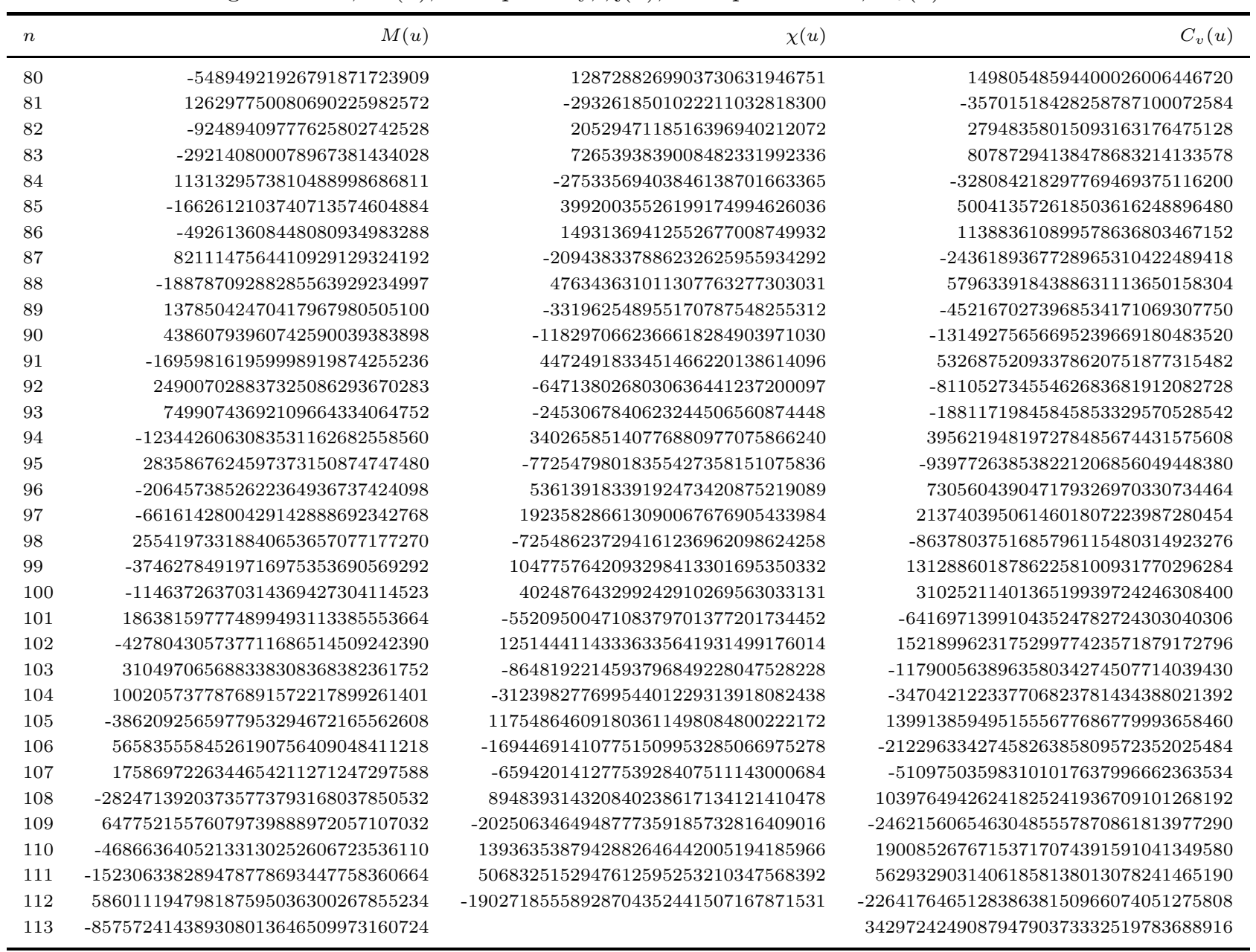

\section{Analysis of the series}

We analysed the series using the same methods as in our previous paper [1] to which we refer the reader for details. Here we will give only a short summary of the results including improved estimates for the critical point and amplitudes. 
The estimates $u_{c}=0.5540663(5)$ for the physical singularity and $\beta=0.12507(2)$ for the critical exponent of the spontaneous magnetisation were obtained from homogeneous differential approximants (which are equivalent to Dlog Padé approximants) by averaging over $[N, M]$ approximants with $|N-M| \leq 1$ using at least 100 series terms. The figure in parenthesis represents the spread among the approximants (basically one standard deviation) and should not be viewed as a measure of the true error as they cannot include possible systematic sources of error. From these estimates it is clear that $\beta=1 / 8$ as expected. However, the estimates converge very slowly towards this value and even with a series as long as the present 114 terms the estimates have not yet settled down to their true value and there is a slight downwards drift in the estimates for both $u_{c}$ and $\beta$. Analysis of the susceptibility and specific heat series yield exponent estimates fully in agreement with the expectations that $\gamma^{\prime}=7 / 4$ and $\alpha^{\prime}=0$. By using our knowledge of the exact values of the critical exponents and assuming that close to $u_{c}$ the estimates for the exponents depend linearly on the estimates for the critical point (inspection of the various approximants clearly supports this assumption) we are led to the improved estimate for the critical point $u_{c}=0.5540653(5)$.

We calculated the critical amplitudes using two different methods, both of which are very simple and easy to implement. In the first method, we note that if $f(u) \sim$ $A\left(1-u / u_{c}\right)^{-\lambda}$, then it follows that $\left.\left(u_{c}-u\right) f^{1 / \lambda}\right|_{u=u_{c}} \sim A^{1 / \lambda} u_{c}$. So we simply form the series for $g(u)=\left(u_{c}-u\right) f^{1 / \lambda}$ and evaluate Padé approximants to this series at $u_{c}$. The result is just $A^{1 / \lambda} u_{c}$. This procedure works well for the magnetisation and susceptibility series (it obviously cannot be used to analyse the specific heat series) and yields the estimates $A_{M}=1.20840(5)$ and $A_{\chi}=0.06172(4)$ where the error bar primarily reflects the uncertainty due to the estimate of $u_{c}$. For the specific heat series two different approaches have been used. In the first approach we look at the derivative of the specific heat series for which the above method should work with $\lambda=1$. This yields the estimate $A_{C}=22.3(1)$. In the second approach we start from $f(u) \sim A \ln \left(1-u / u_{c}\right)$ and form the series $g(u)=\exp (-f(u))$ which has a singularity at $u_{c}$ with exponent $A$. One virtue of this approach is that no prior estimate of $u_{c}$ is needed. However, the spread among estimates from different approximants is quite substantial, though the amplitude estimate is consistent with that listed above. Biasing the estimates at $u_{c}$ yields $A_{C}=22.3(3)$. In the second method, proposed by Liu and Fisher [6], one starts from $f(u) \sim A(u)\left(1-u / u_{c}\right)^{-\lambda}+B(u)$ and then forms the auxiliary function $g(u)=\left(1-u / u_{c}\right)^{\lambda} f(u) \sim A(u)+B(u)\left(1-u / u_{c}\right)^{\lambda}$. Thus the required amplitude is now the background term in $g(u)$, which can be obtained from inhomogeneous differential approximants [5]. This method can also be used to study the specific heat series. One now starts from $f(u) \sim A(u) \ln \left(1-u / u_{c}\right)+B(u)$ and then looks at the auxiliary function $g(u)=f(u) / \ln \left(1-u / u_{c}\right)$. As before the amplitude can be obtained as the background term in $g(u)$. This analysis yields the amplitude estimates listed in table 7 . With 
the exception of the specific heat amplitude there is excellent agreement between these estimates and those obtained from the first method.

Table 7. Estimates for the physical critical amplitudes $A_{M}, A_{\chi}$ and $A_{C}$ from the method of Liu and Fisher obtained from inhomogeneous first-order differential approximants. $\mathrm{L}$ is the degree of the inhomogeneous polynomial.

\begin{tabular}{llll}
\hline$L$ & \multicolumn{1}{c}{$A_{M}$} & \multicolumn{1}{c}{$A_{\chi}$} & \multicolumn{1}{c}{$A_{C}$} \\
\hline 5 & $1.208284(74)$ & $0.061712(47)$ & $20.20(26)$ \\
6 & $1.208270(15)$ & $0.06173(12)$ & $20.13(22)$ \\
7 & $1.208256(80)$ & $0.06170(20)$ & $20.23(23)$ \\
8 & $1.208266(16)$ & $0.06164(21)$ & $20.29(20)$ \\
9 & $1.20824(21)$ & $0.06170(12)$ & $20.28(32)$ \\
10 & $1.208269(35)$ & $0.061866(60)$ & $20.31(26)$ \\
15 & $1.208272(74)$ & $0.06165(15)$ & $20.47(32)$ \\
20 & $1.20824(12)$ & $0.06167(15)$ & $20.464(75)$ \\
25 & $1.208250(47)$ & $0.06172(11)$ & $20.448(46)$ \\
30 & $1.208236(51)$ & $0.06174(20)$ & $20.42(12)$ \\
35 & $1.208228(60)$ & $0.061792(82)$ & $20.40(20)$ \\
40 & $1.208263(70)$ & $0.06159(20)$ & $20.45(12)$ \\
\hline
\end{tabular}

Regarding the value of the confluent exponent $\Delta_{1}$ we have little to add to our previous results. Even with a series as long as 114 terms we could not obtain accurate estimates for $\Delta_{1}$. Again the Baker-Hunter [7] transformed series of the magnetisation favours a value around 1.05 while estimates from the suceptibility series again fall in two groups around 1.15 and 1.4, respectively. Using the transformation of Adler et al 8

$$
G(u)=\lambda F(u)+\left(u_{c}-u\right) \mathrm{d} F(u) / \mathrm{d} u,
$$

where $F(u)$ is the original series and $\lambda$ the leading critical exponent, yields estimates consistent with $\Delta_{1}=1$ for both the magnetisation and susceptibility.

We find a non-physical singularity closer to the origin than $u_{c}$ at $u_{ \pm}=$ $-0.3019395(5) \pm 0.3787735(5)$ with exponents $\beta=-0.1690(2), \gamma^{\prime}=1.1692(2)$ and $\alpha^{\prime}=1.1693(3)$, and a singularity on the negative $u$-axis at $u_{-}=-0.598550(5)$ with exponents equal to those at the physical critical point. Note that our estimate for $\beta$ at $u_{ \pm}$has changed substantially from that given in our previous paper. This is mainly because we have put greater emphasis on estimates obtained from inhomogeneous first and second-order differential approximants. We note that we now have firm evidence to show that the scaling law $\alpha^{\prime}+2 \beta+\gamma^{\prime}=2$ holds at both the physical as well as the non-physical singularities. 


\section{E-mail or www retrieval of series}

The series for the spin-1 Ising model can be obtained via e-mail by sending a request to iwan@maths.mu.oz.au or via the world wide web on http://www.maths.mu.oz.au/ iwan/ by following the instructions.

\section{Acknowledgements}

Financial support from the Australian Research Council is gratefully acknowledged.

\section{References}

[1] Enting I G, Guttmann A J and Jensen I 1994 J. Phys. A: Math. Gen. 276987

[2] De Neef T and Enting I G 1977 J. Phys. A: Math. Gen. 10801

[3] Baxter R J and Guttmann A J 1988 J. Phys. A: Math. Gen. 213193

[4] Enting I G 1978 J. Phys. A: Math. Gen. 11563

[5] Guttmann A J 1989 Phase Transitions and Critical Phenomena vol 13, ed C Domb and J Lebowitz (New York:Academic) pp 1-234

[6] Liu A J and Fisher M E 1989 Physica 156A 35

[7] Baker G A and Hunter D L 1973 Phys. Rev. B 73377

[8] Adler J, Moshe M and Privman V 1981 J. Phys. A: Math. Gen. 172233 